\title{
Micro-Doppler based Target Recognition with Radars: A Review
}

This paper was downloaded from TechRxiv (https://www.techrxiv.org).

\section{LICENSE}

CC BY 4.0

SUBMISSION DATE / POSTED DATE

$07-12-2021 / 10-12-2021$

\section{CITATION}

Hanif, Ali; Muaz, Muhammad; Hassan, Azhar; Adeel, Muhammad (2021): Micro-Doppler based Target Recognition with Radars: A Review. TechRxiv. Preprint. https://doi.org/10.36227/techrxiv.17133611.v1

$\mathrm{DOI}$

10.36227/techrxiv.17133611.v1 


\title{
Micro-Doppler based Target Recognition with Radars: A Review*
}

\author{
Ali Hanif, Muhammad Muaz, Azhar Hasan, and Muhammad Adeel $\dagger$
}

December 7, 2021

\begin{abstract}
With the deployment of radar in versatile scenarios and a wide variety of potential targets, demand for automatic classification of various targets is increasing. The wide variety of radar signatures from physically smaller targets due to lower velocity / radar cross-section thresholds and the increased deployment of radar-based sensors do crowd the radar screen with misinterpreted targets. Micro-Doppler signatures have been widely employed by researchers for the recognition of those targets that exhibit micro-motions. This review article presents the evolution and recent advances in radar micro-Doppler based signature analysis and feature extraction. A review of the micro-Doppler-based target classification techniques along with their applications in defense and commercial sectors, has
\end{abstract}

* "This work has been submitted to the IEEE for possible publication. Copyright may be transferred without notice, after which this version may no longer be accessible."

$\dagger^{\dagger}$ Ali Hanif, Muhammad Muaz, and Azhar Hasan are with the College of Aeronautical Engineering, National University of Sciences and Technology, Islamabad, Pakistan (e-mails: ahanif.ms13ave@student.nust.edu.pk,m.muaz@cae.nust.edu.pk, ahasan@cae.nust.edu.pk).

${ }_{\ddagger}^{\ddagger}$ Muhammad Adeel is with the Department of Electronic and Information Engineering, The Hong Kong Polytechnic University, Hong Kong (e-mail: m.adeel@connect.polyu.hk).

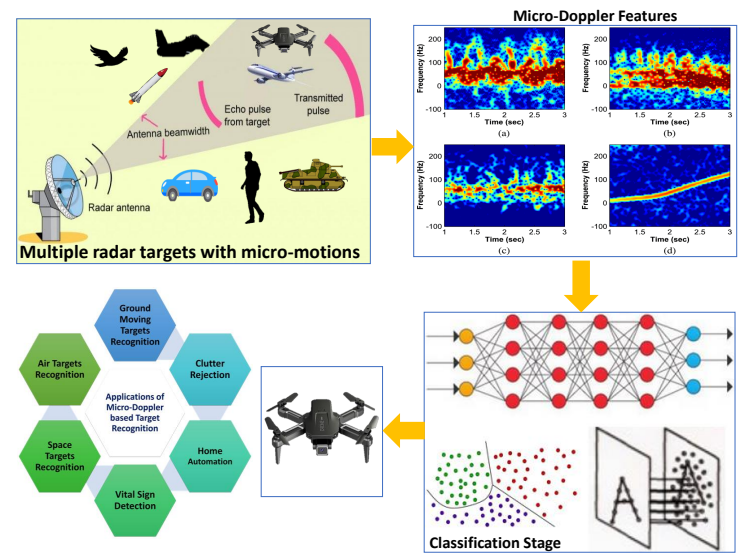


also been presented. This article provides the first review paper in the open literature that systematically covers the major steps along with the adopted practices in micro-Doppler based target recognition. Moreover, the limitations and future trends in the field are also discussed.

Keywords - Micro-Doppler, radar, deep learning, feature extraction, machine learning, pattern recognition, classification algorithms

\section{Introduction}

The use of first operational radar for remote sensing of targets dates back to the second world war in which 'Chain Home' radar network was used by the Royal Air Force to provide early warning about detection of enemy aircraft [1]. The need for automatic target recognition was felt at the earlier stages of radar development and since then it has remained an active area of research. It was as early as 1937 when the target recognition experiments were carried out by adding resonant dipoles to friendly aircraft for their discrimination from enemy aircraft [2]. Secondary Surveillance Radars (SSR) were then introduced to achieve target classification using Identification of Friend and Foe (IFF) transponders. However, this technique was dependent on the target transmitting the required information. In 1980, Merrill Skolnik presented certain approaches in his famous book [3], which can be employed for target recognition. One of the approaches was the Jet Engine Modulation (JEM), which deals with engine induced frequency modulation on the echo signal of radar.

In many scenarios, the sub-components of a target exhibit micro-motions such as rotation, vibration, tumbling, and coning, in addition to the target's "bulk motion". Examples of such micro-motions include rotating blades of a helicopter or multirotor, rotating blades of propeller of a fixed wing aircraft, swinging arms and legs of a pedestrian, and rotation of wind turbines. The micro-motions induce Doppler modulations on the received signal known as micro-Doppler effect [4]. Micro-Doppler effect was first introduced in coherent laser detection and ranging systems [5]. Due to these modulations, sidebands are generated about the target's Doppler frequency shift. The micro-Doppler signature is the characteristic to a particular target's micro-motion and hence it can be exploited for classification of different targets [4]. Figure 1 shows the simulated micro-Doppler signature of a running human with a radial speed of 2 $\mathrm{m} / \mathrm{s}[6]$.

In war time scenarios, rapid and robust target classification is vital as it can help in generating appropriate response according to the type of threat being identified. With the deployment of radars in cluttered urban scenarios and prompt availability of many commercial off-the-shelf (COTS) drones, there is a growing need for detection and classification of smaller targets like humans, drones, bicycles, birds, etc. That is why the velocity and Radar Cross Section (RCS) thresholds need to be lowered for detection of smaller targets. This invites a large number of target signatures, which if not discerned, may lead to loss of situational awareness. Thus, target classification has become critical 


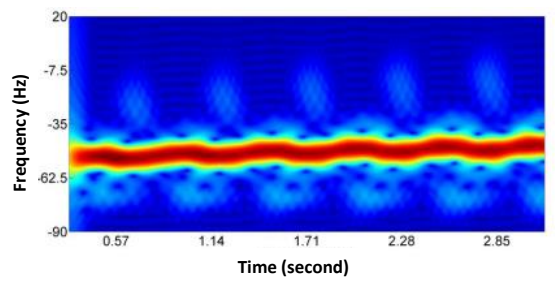

Figure 1: Micro-Doppler signature of a running human [6]. Used under CC license.

for filtering out irrelevant targets [7]. Detection and classification of drones is also crucial in current times for avoiding their misuse for activities like espionage and terrorism. Micro-Doppler signatures have emerged as a useful tool for radar target recognition in such scenarios.

A comparison of our article with existing review articles on the topic [8-12] is presented in Table 1. 
Table 1: COMPARISON WITH EXISTING REVIEW ARTICLES

\begin{tabular}{|c|c|c|c|}
\hline Review Article & Year & Scope of Work & Remarks \\
\hline$[8]$ & 2003 & $\begin{array}{c}\text { Review of mD effect, maths of mD effect, mD signature } \\
\text { analysis and potential applications }\end{array}$ & $\begin{array}{c}\text { No coverage of feature extraction, classification techniques, } \\
\text { datasets and limitations for mD based target recognition }\end{array}$ \\
\hline$[9]$ & 2008 & $\begin{array}{c}\text { Review of mD signature analysis, feature extraction, } \\
\text { applications and future directions }\end{array}$ & $\begin{array}{c}\text { No coverage of classification techniques, datasets and } \\
\text { limitations for mD based target recognition }\end{array}$ \\
\hline$[10]$ & 2014 & $\begin{array}{c}\text { Review of advances in extraction and applications of } \\
\text { radar micro-Doppler signatures }\end{array}$ & $\begin{array}{c}\text { No coverage of feature extraction, classification techniques, } \\
\text { datasets and limitations for mD based target recognition }\end{array}$ \\
\hline$[11]$ & 2015 & $\begin{array}{c}\text { Review of mD effect, mD signature analysis, applications } \\
\text { and future research }\end{array}$ & $\begin{array}{c}\text { No coverage of feature extraction, classification techniques } \\
\text { and datasets for mD based target recognition }\end{array}$ \\
\hline$[12]$ & 2018 & $\begin{array}{c}\text { Review of radar signals in terms of Doppler tolerance, } \\
\text { time-sidelobe level and immunity against jamming }\end{array}$ & $\begin{array}{c}\text { No coverage of mD signature analysis, feature extraction, } \\
\text { classification techniques,applications and datasets for } \\
\text { mD based target recognition }\end{array}$ \\
\hline
\end{tabular}


The remaining paper is arranged as follows: micro-Doppler signature analysis is covered in Section II. Section III covers the micro-Doppler-based feature extraction techniques. Section IV summarizes the micro-Doppler-based classification approaches and dataset generation is covered in Section V. Section VI presents important applications of micro-Doppler-based target recognition. Section VII discusses limitations and possible future directions in the field. The paper is concluded in Section VII.

\section{MICRO-DOPPLER SIGNATURE ANALYSIS}

Fourier transform does not provide time dependent frequency information. Hence, it is not suitable for the analysis of micro-Doppler signals since the spectral content of these signatures vary with time [4]. Time-frequency analysis techniques do provide spectral and temporal information of the micro-Doppler signal simultaneously, in which the following methods are employed [4]:

\subsection{Instantaneous Frequency Analysis}

For a non-stationary signal, instantaneous frequency is defined as:

$$
f(t)=\frac{1}{2 \pi} \frac{d}{d t} \Phi(t)
$$

where $\Phi(t)$ is the phase function of the analytic signal $z(t)=a(t) \exp [\Phi(t)]$. The limitation of this method is that it only provides a single frequency at any time instant. Therefore, it cannot be used for analysis of multi-component signals, like the micro-Doppler signature of a target, containing multiple frequencies at a given time instant. For applying this method on multi-component signals, they need to be decomposed into constituent mono-component signals, using techniques such as Empirical Mode Decomposition (EMD) [13], which can then be analyzed using instantaneous frequency analysis.

\section{$2.2 \quad$ Joint Time-Frequency Analysis}

Joint time-frequency analysis is used for analyzing the time dependent spectral content of micro-Doppler signals. It can be applied to mono-component as well as multi-component signals. Its techniques are classified into linear timefrequency transforms and bilinear time-frequency transforms.

\subsubsection{Linear time-frequency transforms}

Short Time Fourier Transform (STFT) is the commonly used linear time-frequency transform for micro-Doppler signature analysis. STFT is expressed mathematically as follows:

$$
X(\tau, \omega)=\int_{-\infty}^{\infty} x(t) w(t-\tau) \exp (-j \omega t) d t
$$


where $x(t)$ is the signal to be transformed and $w(\tau)$ is the window function. Major pitfall of STFT is that there is a trade-off between time resolution and frequency resolution [14].

\subsubsection{Bilinear time-frequency transforms}

In order to improve the resolution of linear time-frequency transforms and to better analyze the micro-Doppler modulations, bilinear or quadratic timefrequency transforms have been applied. They provide good time-frequency resolution but increase the computational cost and also suffer from the phenomenon of "cross-term interference" [4]. The cross-term gives wrong spectrum distribution and blur the characteristics of the time-frequency signals, thus affecting the physical explanation of bilinear time-frequency transform [15]. Some of the commonly used bilinear time-frequency transforms are listed in Table 2, alongwith their respective kernel functions for reducing the cross-term interference [14]. Any of the bilinear time-frequency transforms can be used for analyzing micro-Doppler signals as long as it satisfies the requirements of high time-frequency resolution and minimum cross-term interference [14]. Other important representations widely used for micro-Doppler signature analysis are Cadence Velocity Diagram (CVD) [16] and Cepstrogram [17], both of which are computed from the spectrogram. The relationship between STFT, spectrogram, CVD and cepstrogram is depicted in Fig. 2.

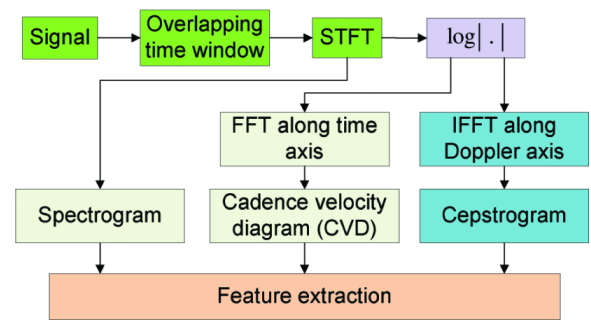

Figure 2: Relationship between STFT, spectrogram, CVD and cepstrogram [18]. (C) 2019 EuMA.

Generally the radar data is first represented in the form of range-Doppler to extract the range and velocity of the targets of interest. Clutter cancellation and detection is performed subsequently. Once the range bins of the targets of interest are identified, then the micro-Doppler signature analysis is done using appropriate time-frequency transforms as discussed earlier. The information from range-Doppler representation can also be used in combination with microDoppler features for target recognition such as in [19], where selected kinematic features extracted from 4-D data matrix (range, azimuth, elevation and Doppler) of a 3-D staring radar are used alongwith micro-Doppler information to train a decision tree. For radars having sufficiently high range resolution (HRR), the displacements caused by the target's micro-motion may become observable from 
the 'macro-range' response. These responses are termed as micro-range signatures [20]. The combination of micro-range and micro-Doppler signatures can be helpful in analysis and recognition of complex motions like human motion where it can be used to isolate the micro-motions from individual body parts leading to improved target detection, classification and discrimination. An algorithm for automated micro-range-micro-Doppler target signature decomposition has been presented in [20]. 
Table 2: BILINEAR TIME-FREQUENCY TRANSFORMS [14]

\begin{tabular}{|c|c|c|}
\hline $\begin{array}{l}\text { Time-Frequency } \\
\text { Transform }\end{array}$ & Mathematical Form & Kernel Function \\
\hline Spectrogram & $|\operatorname{STFT}(\mathrm{t}, \omega)|^{2}$ & - \\
\hline Cohen Class & $\iiint \Phi(\theta, \tau) s(u+\tau / 2) s^{*}(u-\tau / 2) e^{j \theta t-j \Omega \tau-j \theta u} d u d \tau d \theta$ & $\Phi(\theta, \tau)$ \\
\hline Wigner-Ville & $\int s\left(t+\frac{\tau}{2}\right) s^{*}\left(t+\frac{\tau}{2}\right) \exp (-j \omega \tau) d \tau$ & $\Phi(\theta, \tau)=1$ \\
\hline $\begin{array}{l}\text { Pseudo Wigner } \\
\quad(\text { PWD })\end{array}$ & $\int h(\tau) s\left(t+\frac{\tau}{2}\right) s^{*}\left(t+\frac{\tau}{2}\right) \exp (-j \omega \tau) d \tau$ & $\Phi(\theta, \tau)=h(\tau)=\exp \left\{j \alpha \tau^{2} / 2\right\}$ \\
\hline $\begin{array}{l}\text { Smooth Pseudo } \\
\text { Wigner-Ville }\end{array}$ & $\int s(t-u) \operatorname{PWD}(\mathrm{t}, \omega, \alpha) \mathrm{du}$ & $\Phi(\theta, \tau)=h(\tau)=\exp \left\{j \alpha \tau^{2} / 2\right\}$ \\
\hline Choi-Williams & $\iint K_{C W}(u-t, \tau) s\left(u+\frac{\tau}{2}\right) s^{*}\left(u+\frac{\tau}{2}\right) e^{-j \omega \tau} d u d \tau$ & $\Phi(\theta, \tau)=K_{C W}(\theta, \tau)=\frac{\exp \left\{-\theta^{2} \tau^{2} / \sigma\right\}}{4 \pi^{3 / 2} \sqrt{\tau^{2} / \sigma}}$ \\
\hline Cone Kernel & $\iiint K_{C K}(t-u, \tau) s\left(u+\frac{\tau}{2}\right) s^{*}\left(u+\frac{\tau}{2}\right) e^{-j \omega \tau} d u d \tau$ & $\Phi(\theta, \tau)=K_{C K}(t, \tau)=\left\{\begin{array}{cc}g(\tau) ; & |t / \tau|<1 / 2 \\
0 ; & |t / \tau|>1 / 2\end{array}\right.$ \\
\hline
\end{tabular}




\section{FEATURE EXTRACTION FROM MICRO- DOPPLER SIGNATURES}

To perform target recognition based on the micro-Doppler effect, certain discriminating features are usually extracted. The accuracy of the classifier is dependent on the robustness of the selected features and its relevance to the dataset. Micro-Doppler returns from a target depend on its RCS, radar operating frequency, and aspect angle. Therefore, extracted features should be robust enough against variations caused by these factors. Feature extraction for microDoppler-based classification can be divided into two categories depending upon the type of classifier employed.

- First is to directly use the micro-Doppler signature images as features for classification. These images can be in the form of spectrograms [21-23], CVD [24], cepstrograms or a combination of these micro-Doppler signature images [24]. This is particularly used when Deep Convolutional Neural networks (DCNNs) are employed as the classifier [21-25].

- Second approach is to extract statistically uncorrelated or independent features from micro-Doppler signature using methods like Singular Value Decomposition (SVD) [26,27], Principal Component Analysis (PCA) [28] and Independent Component Analysis (ICA) [29]. Empirical Mode Decomposition (EMD) has also been successfully employed for extracting micromotion features generated by rotating or vibrating structures [30-32]. An algorithm proposed in [7], extracts four micro-Doppler features from spectrograms and cepstrograms, to discriminate between UAVs and birds. Cadence Velocity Diagram Frequency Profile (CVDFP) and Mean CVD are used as features for micro-Doppler classification in [28,33] respectively.

The above mentioned methods decompose micro-Doppler signature into components that are uncorrelated or independent, but they are not necessarily linked to motion of individual body parts. In the case of complex motion like human walking or running, the first step is to decompose the micro-Doppler signature into components related to the individual body parts or structures [4]. Such a methodology is proposed in $[34,35]$. However, it covered signature decomposition for human walking and running only. In the same context, Viterbi algorithm is also applied for decomposition of micro-Doppler signatures and estimation of hidden state in joint time-frequency domain [36].

Table 3 lists down the features employed ('Features' column) in a variety of works on $\mathrm{mD}$ radar target recognition. The above listed feature categories are also evident from the information presented in Table 3. Feature extraction is done automatically in the case of deep learning classifiers such as CNNs. However, manual feature extraction has to be done for conventional classifiers such as SVM and Naïve Bayes. Manual feature extraction offers more control over the classification process and can prove beneficial in certain scenarios where we want to give more weight to particular features. On the other hand, automatic feature extraction by deep learning classifiers may extract certain important 
features that may otherwise be missed during the manual feature extraction. Important features used for classification of birds and drones are $\mathrm{mD}$ periodicity, spectrum width and symmetry [37]. mD periodicity represents the rotation rate, which will be higher in case of drones as compared to birds. The wing beat frequency of birds is between 2 and 20 beats per seconds [37]. $\mathrm{mD}$ spectrum width represents the maximum velocity of micro-motions relative to main velocity, which is higher in case of drones. Spectrogram symmetry can also be used for differentiating between birds and drones (with even number of blades) as birds have an asymmetric spectrogrm [37]. Other discriminating features are RCS and target velocity but their dependence on aspect angle and radar frequency makes them less robust [37]. Important features used for classification of humans and human gait are torso Doppler frequency and bandwidth, total Doppler bandwidth, offset of the total Doppler, Doppler bandwidth without $\mathrm{mD}$ effect, period of limb motion, average radial velocity and normalised standard deviation of Doppler signal strength $[38,39]$. These features are extracted

from the $\mathrm{mD}$ signature by computing the mean, minimum and maximum of lower and upper envelopes of spectrogram, mean Doppler, torso bandwidth, total bandwidth and outer bandwidth $[38,40]$. Feature extraction is peculiar to problem and depends on factors such as the environment, type of radar and characteristics of target. For reliable target recognition, the selected features (i) should provide information about target parameters such as rotation rate, blade flash frequency and number of rotor blades (ii) should be discriminative between concerned target classes e.g., the bulk velocity can be a discriminative feature for classifying between drone and non-drone but it can't be for mini-UAV and birds as both have velocities in the same range [37]. The discrimination between the classes can also be quantitavely verified by separability measures like Jefferies-Matusita distance, Transformed Divergence, etc. (iii) should be robust with respect to target type, radar settings, background environment and measurement parameters such as operating frequency, aspect angle and polarization [37].

\section{MICRO-DOPPLER-BASED TARGET CLAS- SIFICATION ALGORITHMS}

For classification of different radar targets based on their micro-Doppler features, different types of classification algorithms have been used in the open-literature. These approaches can be broadly classified into model-based and data-driven methods $[41,42]$.

\subsection{Model-based Classification Approach}

Model-based methods rely on the parametric models of the dynamic and kinematic properties of targets for classification. Examples of such models are the Thalmann model for human walking [43], and Vignaud model for human running [44]. These methods estimate the probability of radar data to fit a prede- 
fined model by simulating the data with different input parameters of the model, and find out the parameters that minimize the difference between the simulated and real data. In [45], a model-based classification of human motion is proposed by applying particle filtering to micro-Doppler spectrum of targets. Particle filters are a set of Monte Carlo algorithms, that use a set of particles with associated weights to represent the required posterior density and to compute estimates. Model-based classification methods can provide useful information regarding the current dynamic state of target by providing estimates of target's motion parameters. However, these methods require high processing power due to model calculations for different parameters, generalized models, and tackling the problem of non-convergence for large number of parameters [42].

\subsection{Data-driven Classification Approach}

Data-driven classification methods can be further categorized into template matching, conventional Machine Learning (ML) techniques and Deep Learning (DL) techniques.

\subsubsection{Template Matching}

Template matching methods perform classification by matching the input data to a signature database or reference library. The library contains the reference signatures of all possible target classes. A class is assigned based on the confidence level of its matching with a certain reference signature. In [46, 47], micro-Doppler classification has been achieved using Dynamic Time Warping (DTW), which is a template-based classification technique. DTW is a technique used in speech recognition and it can measure the similarity between two time series with varying speeds. In performance comparison with k-Nearest Neighbors (k-NN), another template-based technique, has also been provided in [47]. k-NN classifier is also used in $[29,48]$ for radar micro-Doppler-based target classification. These methods are simpler to implement as compared to model-based and supervised learning techniques. However, for large databases, these methods can be time-consuming as the input data has to be compared with all possible entries in the reference library. Moreover, larger libraries also require more memory as these are needed during classification, in contrast to supervised learning, where training data is used offline.

\subsubsection{Conventional ML Classification Algorithms}

Conventional ML classifiers that have been employed for micro-Doppler-based classification include linear and non-linear Support Vector Machines (SVM) [28, $32,33,48,49]$, naïve Bayes [49,50], Maximum A Posteriori (MAP) [7], subspace reliability analysis [51], discriminant analysis [52], and decision trees [19,53]. These classifiers have achieved good results with classification accuracies of $90 \%$ and above on their acquired datasets under different experimental scenarios. The advantages of these conventional ML techniques over deep learning are the 
requirement of less amount of training data, especially if the dimension of the feature space can be reduced using signal processing techniques such as SVD or PCA [41]. The overall computational complexity of these classifiers is less than deep learning based classifiers, thus they are much faster and less complex. A comparison of both types of classifiers in terms of computational complexity is provided in [54]. The total time for the SVM classifier is reported to be 99 seconds, whereas it is 157 seconds and 241 seconds for a CNN and convolutional autoencoder, respectively.

\subsubsection{Deep Learning Classification Algorithms}

Deep learning (DL) based classification approaches have certain inherent advantages over conventional ML approaches. They are less dependent on domain knowledge as they can automatically extract the features as well as classification boundaries from the micro-Doppler signatures [22,24]. Due to the elimination of feature extraction step, they can save the processing time required by feature extraction algorithms [21] as well as avoid human errors in making the right choices. Deep learning classifiers are also reported to learn the clutter and noise patterns during training e.g., during the convolution filtering process in CNNs. Clutter has a significant impact on the performance of other classifiers, in which such deep learning architectures may be useful by the automatic capability of clutter cancellation $[40,54]$. Moreover, deep learning architectures can extract certain important features, which may otherwise be missed by the manual feature extraction process in case of ML based approaches [22].

Deep Convolutional Neural Networks (DCNNs) have been applied for radar micro-Doppler-based classification [21-25]. However, DCNNs have certain drawbacks: they require input data in the form of images and require diverse training sets for better classification. As the input to DCNNs is in the form of micro-Doppler signature images, accuracy can reduce if there are variations in micro-Doppler signature due to irregularities in the micro-motion dynamics of a target [22]. Moreover, the training and validation process needs to be rigorous and diverse for better classification and to avoid overfitting [21].

In addition to DCNNs, Recurrent Neural Networks (RNNs) have also been exploited for target classification based on micro-Doppler effect [55-59]. Out of different RNN types, Long Short-Term Memory (LSTM) networks are popular, as they can overcome the issue of vanishing / exploding gradient problem [60] and are able to learn both long and short data sequences compared to other RNN architectures [61]. Use of RNNs bring the advantages of classifying measurements with variable observation time and can also deal with measurements that include transitions between classes over time.

A deep Convolutional Autoencoder (CAE), that essentially combines the benefits of Autoencoder and CNN, is proposed in [54] to classify 12 types of aided and non-aided human activities with an accuracy of $94.2 \%$. Researchers have also employed hybrid classifiers for micro-Doppler-based target classification, that combine both the conventional ML and DL classifiers to take advantages of both techniques [62]. 


\section{DATASET GENERATION FOR DATA-DRIVEN ALGORITHMS}

Any coherent Doppler radar such as pulse-Doppler, Continuous Wave (CW), or Frequency-Modulated Continuous Wave (FMCW) can be employed for capturing micro-Doppler returns from the targets. CW radar offers advantages of high sampling rates and longer integration times whereas pulse-Doppler / FMCW radar can calculate target range; thus improving the Signal to Noise Ratio (SNR) of micro-Doppler returns. However, pulse-Doppler and FMCW radars have relatively low Pulse Repetition Frequency (PRF) / Scan Repetition Frequency (SRF), which can result in major Doppler fold-over [63].

Collecting real data for the radar micro-Doppler-based target recognition is laborious and expensive. However, diversity in the limited training dataset can be enhanced by generating additional data with Generative Adversarial Networks (GANs) and model-based simulations [64]. Additionally, transfer learning and unsupervised pre-training methods can also be used in case of low training sample support, thus preventing the models from over-fitting $[64,65]$. GANs have been used in the literature for generating additional training samples from the actual data because they have the ability to synthesize data whose distribution is very close to the real-world datasets $[69,72,73]$. However, the number of GANs being used increase with the increase in target classes, and training of large number of GANs is challenging [69]. Transfer learning has been employed for micro-Doppler-based classification using DCNNs [21,24,65]. In [65], unsupervised pre-training is implemented through the use of convolutional autoencoder and a comparison with the transfer learning approach is presented. Table 3 presents a summary of various types of datasets, classification methodologies and features adopted in the literature for micro-Doppler-based target recognition.

An important practical issue arises when all the radar data is not available beforehand for training the model, rather data is arriving sequentially as learning continues. In such scenarios, if the model is retrained from scratch every time on the arrival of new data, it can result in huge computational cost. On the other hand, if the model is simply updated with new data, it can result in catastrophic forgetting of past learned tasks [74]. A comparative study of continual learning methods for micro-Doppler-based human activity classification has been presented for the first time in [75]. The authors have considered two continual learning scenarios of domain incremental learning (DIL) and class incremental learning (CIL), for comparing the performance of regularization- [76-78] and exemplar-memory-based continual learning methods [79-81]. 
Table 3: COMPARISON OF DATASETS, CLASSIFICATION APPROACHES AND FEATURES USED FOR MICRO-DOPPLER BASED TARGET RECOGNITION

\begin{tabular}{|c|c|c|c|c|c|c|c|c|}
\hline References & Year & Radar & $\begin{array}{l}\text { Classification } \\
\text { Approach }\end{array}$ & $\begin{array}{l}\text { Target } \\
\text { Classes }\end{array}$ & Features & $\begin{array}{l}\text { Samples per } \\
\text { Target Class }\end{array}$ & $\begin{array}{c}\text { Environmental } \\
\text { Scenario }\end{array}$ & \begin{tabular}{|l|} 
Classification \\
Accuracy (\%)
\end{tabular} \\
\hline [49] & 2013 & $\mathrm{CW}$ & $\begin{array}{c}\text { Linear SVM/ } \\
\text { Nonlinear SVM/ } \\
\text { Naïve Bayes }\end{array}$ & $\begin{array}{l}\text { Birds, Stationary Rotor, } \\
\text { Mini Quadcopter, } \\
\text { Mini Helicopter, } \\
\text { Mini Fixed-Wing Plane }\end{array}$ & $\begin{array}{c}\text { Transformed } \\
\text { Eigenvectors } \\
\text { and Eigenvalues }\end{array}$ & $7500^{\mathrm{a}}$ & Outdoor & \begin{tabular}{|c|}
$94.91 / 95.39$ \\
$/ 93.6$ \\
\end{tabular} \\
\hline [66] & 2013 & $\begin{array}{c}\text { Pulse } \\
{[67]}\end{array}$ & Nonlinear SVM & \begin{tabular}{|c|} 
Human Walking (different \\
speeds), Horse with Rider \\
(different speeds), Both
\end{tabular} & \begin{tabular}{|c|} 
SFP, CVDFP $^{c}$, \\
SFP-CVDFP-PCA \\
\end{tabular} & $\begin{array}{l}28,112 \text { (For } \\
\text { last class only) }\end{array}$ & Outdoor & $>90$ \\
\hline$[7]$ & 2015 & $\mathrm{CW}$ & Min Error Rate & \begin{tabular}{|l} 
Large Man-made, Bio Life, \\
Small Multicopter, Small \\
Heli, Small Fixed-Wing \\
\end{tabular} & \begin{tabular}{|c|} 
Mean RCS, \\
Target Velocity, \\
mD Periodicity and \\
Spectrum Width \\
\end{tabular} & $\begin{array}{l}\text { Hand-drawn } \\
\text { likelihood } \\
\text { curves used }\end{array}$ & Outdoor & Not Provided \\
\hline [68] & 2015 & $\mathrm{CW}$ & $\begin{array}{c}\text { Nä̈ve Bayes / } \\
\text { Shape Spectrum }\end{array}$ & Humans & $\begin{array}{l}\text { Shape spectrum } \\
\text { features based } \\
\text { on CVD }\end{array}$ & 240 & Indoor & $>90$ \\
\hline [40] & 2015 & Pulse & $\begin{array}{l}\text { Two-Nearest } \\
\text { Neighbor }\end{array}$ & Human Activity & \begin{tabular}{|c|} 
Mean,min and max \\
of lower/upper spec \\
-trogram envelope, \\
Mean doppler,Torso, \\
total and outer BW \\
\end{tabular} & 1680 & Indoor & $>88$ \\
\hline [22] & 2016 & $\mathrm{CW}$ & DCNN & $\begin{array}{l}\text { Human, Dog, Horse, } \\
\text { Car / Human Activity }\end{array}$ & \begin{tabular}{|l|} 
Automatic feature \\
extraction by CNN \\
from spectrograms \\
\end{tabular} & $40 / 144$ & Outdoor & $97.6 / 90.3$ \\
\hline [33] & 2017 & $\mathrm{CW}$ & SVM & $\begin{array}{c}\text { Drones, } \\
\text { Birds (Simulated) }\end{array}$ & \begin{tabular}{c|} 
Mean $\mathrm{mD}$ \\
spectrogram, Mean \\
CVD, 1st left \\
singular vector \\
\end{tabular} & 80 & $\begin{array}{l}\text { Outdoor } \\
\text { (for Drones only) }\end{array}$ & $96-100$ \\
\hline [24] & 2017 & FMCW & GoogLeNet CNN & Drones & \begin{tabular}{|c|} 
Automatic feature \\
extraction by CNN \\
from merged images
\end{tabular} & \begin{tabular}{|l|}
50,000 (Chamber) \\
10,000 (Outdoor)
\end{tabular} & $\begin{array}{c}\text { Anechoic Chamber/ } \\
\text { Outdoor }\end{array}$ & $94.7 / 100$ \\
\hline [55] & 2017 & $\mathrm{CW}$ & LSTM & Upto 3 Human Gaits & $\begin{array}{l}\text { Automatic feature } \\
\text { extraction by RNN } \\
\text { from spectrograms } \\
\end{array}$ & $3235-9889$ & Outdoor & 89.1 \\
\hline [32] & 2018 & $\mathrm{CW}$ & Nonlinear SVM & $\begin{array}{l}\text { Birds, Stationary Rotor, } \\
\text { Mini Quadcopter, } \\
\text { Mini Helicopter, } \\
\text { Mini Fixed-Wing Plane } \\
\end{array}$ & \begin{tabular}{|c|}
08 statistical and \\
geometrical features \\
extracted \\
from IMFs
\end{tabular} & $1129-3617$ & Outdoor & $>90$ \\
\hline [61] & 2018 & FMCW & $\begin{array}{c}\text { CNN / Residual } \\
\text { Network / CNN- } \\
\text { LSTM } \\
\end{array}$ & $\begin{array}{c}\text { Single and Two Person, } \\
\text { Bicycle, Car }\end{array}$ & $\begin{array}{c}\text { Automatic feature } \\
\text { extraction by } \\
\text { CNN / RNN } \\
\text { from spectrograms }\end{array}$ & $\begin{array}{l}60 \text { and } 44, \\
22,60\end{array}$ & Outdoor & \begin{tabular}{|l|}
$98-100$ (Two \\
Class), $79-93$ \\
(Three Class) \\
\end{tabular} \\
\hline [54] & 2018 & $\mathrm{CW}$ & $\begin{array}{c}\text { Convolutional } \\
\text { Autoencoder (CAE) }\end{array}$ & $\begin{array}{l}12 \text { Human } \\
\text { Activities }\end{array}$ & $\begin{array}{l}\text { Automatic feature } \\
\text { extraction by CAE } \\
\text { from spectrograms } \\
\end{array}$ & $50-149$ & Indoor & 94.2 \\
\hline [25] & 2020 & FMCW & Light CNN & Drones, Noise & $\begin{array}{c}\text { Automatic feature } \\
\text { extraction by CNN } \\
\text { from improved } \\
\text { spectrograms } \\
\end{array}$ & 700 & Outdoor & 97.14 \\
\hline [69] & 2020 & $\mathrm{CW}$ & $\begin{array}{l}\text { DCNN-6 and GANs / } \\
\text { AlexNet and VGG-16 }\end{array}$ & Human Activity & $\begin{array}{l}\text { Automatic feature } \\
\text { extraction by CNN } \\
\text { from spectrograms }\end{array}$ & $\begin{array}{c}144 \text { (Actual) }+ \\
1440 \text { (Using } \\
\text { GANs) }\end{array}$ & Outdoor & $97.6 / 95.56$ \\
\hline [62] & 2020 & FMCW & Hybrid SVM-CNN & Human, Vehicle & $\begin{array}{c}\text { Range extension/ } \\
\text { variance, Velocity, } \\
\text { Velocity ext/var } \\
\end{array}$ & $\begin{array}{c}2500 \text { (Vehicle) } \\
500 \text { (Human) }\end{array}$ & Outdoor & 96 \\
\hline [70] & 2020 & $\mathrm{CW}$ & $\begin{array}{c}\text { Dictionary } \\
\text { Learning }\end{array}$ & $\begin{array}{c}\text { Mini Helicopter, } \\
\text { Quadcopter and } \\
\text { Hexacopter (Single } \\
\text { and Multiple Targets) }\end{array}$ & 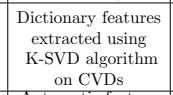 & 200 & Indoor & 93.38 \\
\hline$[21,71]$ & 2020 & FMCW & $\begin{array}{l}\text { GoogLeNet CNN / } \\
\text { Series Network }\end{array}$ & $\begin{array}{l}\text { Drone, Birds, } \\
\text { Clutter, Noise }\end{array}$ & $\begin{array}{l}\text { Automatic feature } \\
\text { extraction by CNN } \\
\text { from spectrograms }\end{array}$ & 600 & Outdoor & $99 / 94.4$ \\
\hline [19] & 2020 & Pulse & $\begin{array}{c}\text { Two Stage } \\
\text { Decision Tree }\end{array}$ & $\begin{array}{c}\text { Drone, } \\
\text { Non-Drone }\end{array}$ & \begin{tabular}{|c|} 
RCS, Velocity, \\
Height, Kinematic \\
and $\mathrm{mD}$ information \\
\end{tabular} & $\begin{array}{c}4622 \text { (Drone) } \\
9244 \text { (Non-Drone) }\end{array}$ & Outdoor & \begin{tabular}{|c|}
$85.02\left(\mathrm{TPR}^{\mathrm{e}}\right)$ \\
$0.37\left(\mathrm{FPR}^{\mathrm{f}}\right)$
\end{tabular} \\
\hline
\end{tabular}




\section{APPLICATIONS AND PRACTICAL IMPLE- MENTATIONS}

Micro-Doppler-based target recognition has found applications in a variety of defense as well as commercial fields. Some important areas of application are depicted in Fig. 3.

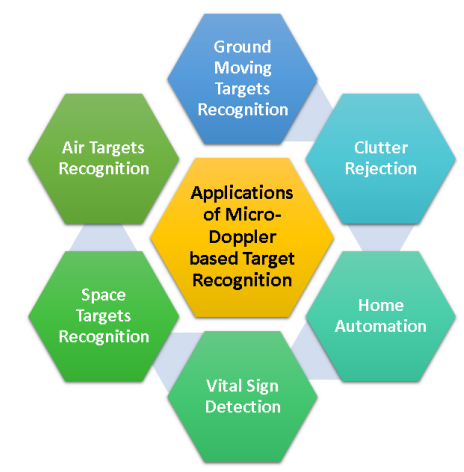

Figure 3: Applications of micro-Doppler-based target recognition.

\subsection{Ground Moving Targets Recognition}

Classifying ground moving objects such as humans, roadway vehicles, and animals are important for applications like perimeter security, intelligent transportation system, surveillance, human gait analysis, etc. Micro-Doppler effect has been successfully employed for recognition of ground moving targets. In [22], four types of ground moving targets were classified based on micro-Doppler spectrogram images using DCNN. In addition to target recognition, seven types of human activities were classified such as walking, running, crawling, and boxing. The authors achieved classification accuracies of $97.6 \%$ and $90.9 \%$ for target recognition and human activity classification respectively on their acquired datasets. Figure 4 shows the extracted spectrogram images of four types of ground targets from [22] whereas spectrograms of four human activities are shown in Fig. 5 [22].

Six different types of pedestrian movements have been classified in [82] using a Support Vector Machine (SVM) classifier; the dimensions of which were reduced using Principal Component Analysis (PCA) applied on "aligned" spectrograms. Classification of ground vehicles, as wheeled or tracked vehicles, has been done in [27]. The classification was performed using an SVM classifier based on micro-Doppler features and SVD. Micro-Doppler-based target recognition has also found applications in the field of automotive industry and road safety. An approach for joint estimation of tracks and micro-Doppler signatures in a real-time multi-target scenario has been presented in [83]. The authors have proposed to use the algorithm for driver assistance to avoid crashes with 

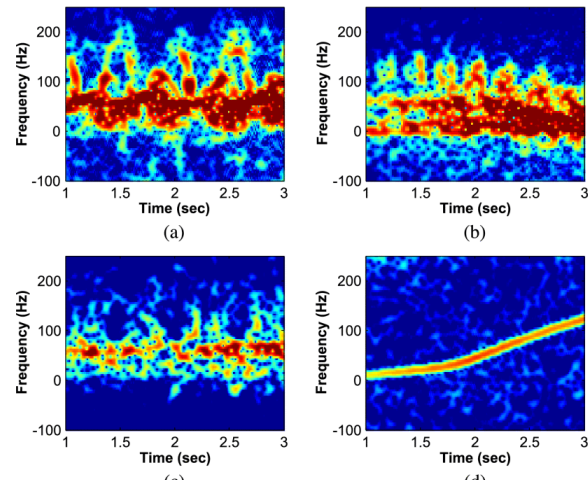

Figure 4: Sample spectrograms from [22]. (a) Human. (b) Dog. (c) Horse. (d) Car. (C)2016 IEEE.
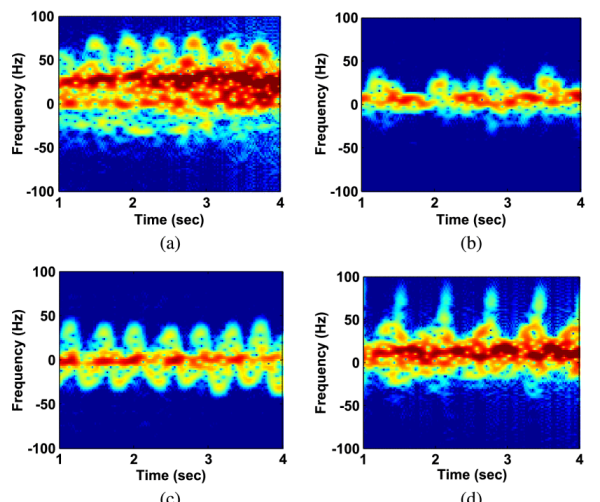

Figure 5: Sample spectrograms of four human activities from [22]. (a) Running. (b) Crawling. (c) Boxing still. (d) Boxing forward. (C)2016 IEEE. 
other vehicles and obstacles, for intelligent traffic light systems to monitor traffic participants at pedestrian crossings, and for intelligent street light systems.

Micro-Doppler-based target recognition have also been used for perimeter security and surveillance of critical infrastructures such as military bases, airports, dams and grid stations $[83,84]$. It has the potential to replace infrared-based motion sensors, which have a high false alarm rate because the infrared sensors can create alarms even for moving foliage and roaming animals. For perimeter security, the humans, vehicles, and animals are automatically differentiated [22].

\subsection{Air Target Recognition}

Primary purpose of radars, which also triggered their invention, is the detection of air targets such as aircraft, helicopters, and drones. Recognition of air targets can give an edge to military forces over their adversary. Incorrect classification of air targets can result in friendly fires and can even change the outcome of a conflict.

Micro-Doppler effect can be used for recognition of air targets as these targets possess micro-motion dynamics such as rotation of propellers of fixed wing aircraft, rotation of fan and turbine in case of jet engines, rotation of helicopter blades, and rotation of rotors in case of drones [4]. Plenty of research has been done for recognition of drones based on micro-Doppler effect [7,19,23,25,30-32]. Micro-Doppler-based target recognition is also employed for classification of loaded and unloaded drones [50], to differentiate birds from drones [21,49], and for classification of propeller based aircraft [85]. Micro-Doppler signatures from helicopter rotor blades have been studied in [86-88]. The research in this field has also resulted in useful commercial products for drone surveillance and security, and for unmanned air system traffic management [89]. One such product, named "Gamekeeper 16U" [89], used for detection, tracking, and classification of Unmanned Air Systems (UAS) is shown in Fig. 7a. It is currently deployed at various airports as part of their counter-UAS strategies for ensuring security and aviation safety [89]. A two stage decision tree approach has been proposed in [19] to classify drones from other confuser targets using the Gamekeeper $16 \mathrm{U}$ radar. Sample spectrogram of DJI Inspire I quadcopter obtained with the Gamekeeper 16U radar is shown in Fig. 6.

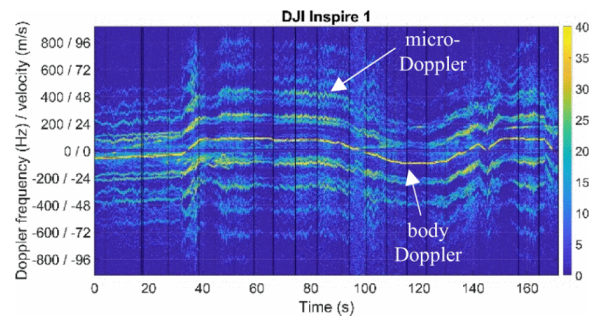

Figure 6: Example spectrogram from DJI Inspire 1 with the Gamekeeper staring radar. [19]. (C)2020 IEEE. 


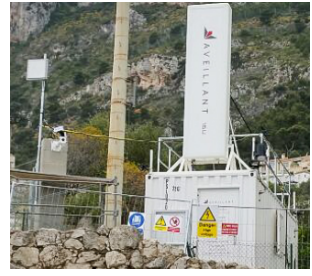

(a) Aveillant's Gamekeeper $16 \mathrm{U}$ counterUAS radar [89].

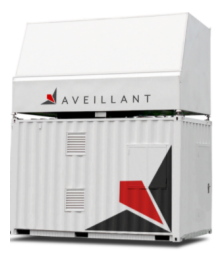

(b) Aveillant's Theia 16A wind farm tolerant radar [89].

Figure 7: Aveillant's Gamekeeper 16U and Theia 16A commercial radars using micro-Doppler phenomenon [89].

\subsection{Clutter Rejection from Wind Turbines}

Countries around the world are installing large number of wind farms to generate clean and green electricity. However, these wind farms are a major source of clutter for nearby radars due to their large RCS and time varying Doppler return. A typical wind turbine could have a RCS of the order of $60 \mathrm{dBsm}$ at Xband [90]. The impact of such wind farm clutter on the performance of aviation radars was studied in [91]. Negative effect of wind turbine clutter on weather radars has also been reported in $[92,93]$. Due to their time varying Doppler return, wind turbine clutter cannot be mitigated using classic ground clutter cancellation techniques [94].

Micro-Doppler signature of wind turbines have been studied in [95, 96]. Micro-Doppler signature identification in the presence of wind turbine clutter for airborne pulse-Doppler radar is presented in [97]. Mitigation of the radar imprints from wind turbines using a combination of CNN and Multilayer Perceptron (MLP) is presented in [98]. The classification of wind turbines was done based on target's attributes and high resolution Doppler spectrum. A commercial radar, named "THEIA 16A", designed by Aveillant (Thales company) for wind farm clutter mitigation is shown in Fig. 7b. It uses the micro-Doppler effect to classify between aircraft and wind turbines, and can present an uncluttered picture of airspace to air traffic controllers by minimizing the number of false tracks.

\subsection{Space Targets Recognition}

Distinctive micro-Doppler signatures result from various types of micro-motions exhibited by space targets. These micro-motions include precession, nutation, spinning, and wobbling. Micro-motion parameters such as spin rate, precession rate, inertia ratio, and nutation angle can be extracted from the micro-Doppler signatures of these space targets, which can be used for their classification. Distinction of a ballistic missile warhead from other confusing space targets is paramount because of higher cost of interceptors and minimum reaction time [99]. Tactical Ballistic Missile (TBM) has to be intercepted during its mid-course 
phase. However, in this phase, separation of multiple boosters from the missile takes place, which results in interfering targets for classification. Warhead of a ballistic missile undergoes precession and nutation motions whereas wobbling motion is exhibited by a decoy. This difference is depicted in their respective micro-Doppler signatures and can be employed to discriminate between ballistic missiles and decoys. In [99], authors have presented an approach based on microDoppler effect for classification of space targets into warhead and confusing target classes. The two target classes are shown in Fig. 8. Micro-Doppler signature extraction from ballistic targets is considered in [100]. Micro-Doppler signature resulting from precession motion of warhead is shown in Fig. 9. Other works on micro-Doppler-based recognition of ballistic targets include [101-107].
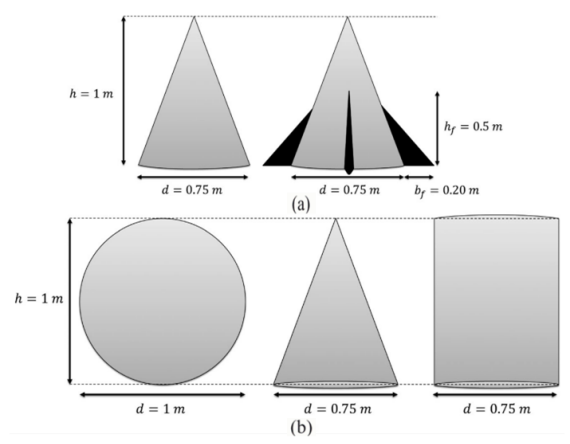

Figure 8: Two target classes along with their subclasses. (a) Warheads. (b) Confusing objects. [99]. Used under CC license.

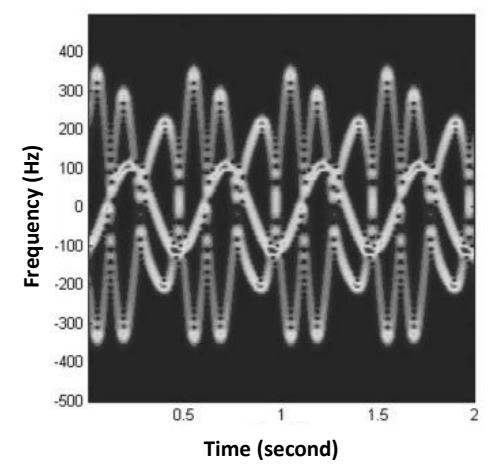

Figure 9: Precession induced micro-Doppler signature [100]. C2010 IEEE. 


\subsection{Home Automation}

Concept of smart homes is gaining popularity with the evolution of Internet of Things (IoT). One aspect of home automation is security such as access control and alarm systems, where the micro-Doppler-based target recognition offers a promising solution. Gesture recognition and control can also be achieved using micro-Doppler features. Radars do not require any tag attached to human hand, can penetrate through materials, and are more robust to variations in light, distance, etc. [4]. Hand gesture recognition based on micro-Doppler signatures has been covered in [108-112].

Another aspect of home automation is the indoor monitoring of inhabitants, especially those who have a medical condition to cater for any health emergency situation. As per the statistics of U.S. Center for Disease Control and Prevention (CDC), the death rate in older adults increased by 30\% from 2007 to 2016 [113]. Radar-based human gait monitoring offers certain advantages like it is contactless and it doesn't violate the privacy of an individual. Micro-Doppler effect has been exploited for monitoring of abnormal human activities inside homes, offices, etc. [4]. Human motion consists of periodic movements such as walking, running, and non-periodic movements such as falling, kneeling. These aperiodic movements can be an indication of a health related emergency such as heart attack, unconsciousness or a severe fall. Analysis of micro-Doppler signatures of various human body parts can give an indication of such situations and can be used for indoor monitoring of habitats $[39,114,115]$. Spectrograms of various human motions used for fall detection in [116] are shown in Fig. 10. Micro-Doppler signatures have been used in [117] for in-home aided and unaided gait recognition with multiple radar and sonar systems.


Figure 10: Spectrograms of four human motions [116]. (a) Fall. (b) Sit. (c) Bend. (d) Walk. (C)2018 IEEE.

Subspace classification of five types of human gaits using radar micro-Doppler signatures have been presented in [118]. These gaits include normal walking, limping with one leg, limping with both legs, cane-assisted synchronized walk and cane-assisted unsynchronized walk. Few sample spectrograms of these gaits 
from [118] are shown in Fig. 11.

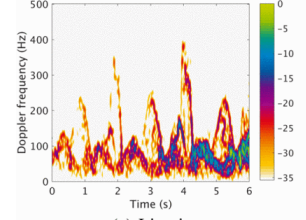

(a) Limping

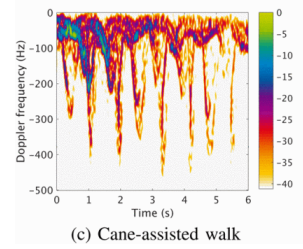

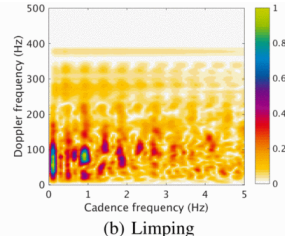

(b) Limping

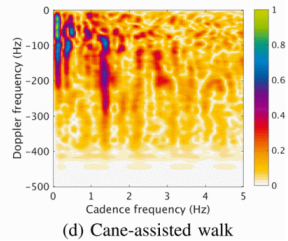

Figure 11: Spectrograms and cadence velocity diagrams of different types of human gaits from [118]. (C)2018 IEEE.

\subsection{Vital Sign Detection}

Vital signs, such as heart rate and respiratory rate, are important indications of human health. These signs are also being used in search and rescue operations for detection of survivors trapped in rubble. Micro-motions resulting from heartbeat and breathing have distinctive micro-Doppler signatures, which can be used for vital sign detection of humans [119-123]. Figure 12 shows the micro-Doppler signature for respiration of a walking human obtained using an ultra wideband radar [124].
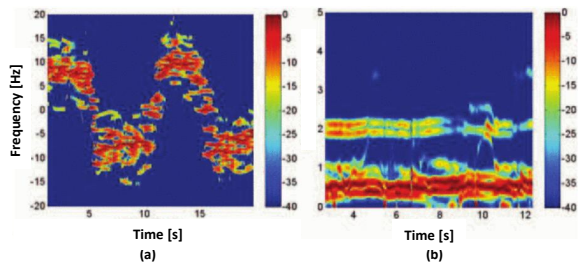

Figure 12: (a) Spectrogram of human walking towards and away from radar. (b) Extracted micro-Doppler trajectory of respiration [124]. (C)2016 IEEE.

\section{LIMITATIONS AND FUTURE TRENDS}

There are research avenues related to micro-Doppler-based target recognition that needs further exploration. In addition to this, certain limitations also exist. 
One of the important limitations for micro-Doppler-based radar target recognition is that there is no widely-accepted open-source benchmark dataset that can be used to check the evolution of micro-Doppler based target recognition methodologies. As discussed in Section 5, researchers have developed and used their own datasets for testing the classification performance of their proposed algorithms. However, each dataset is different in terms of type of radar used, number and type of targets considered, diversity (in terms of changing frequency, aspect angle, polarization etc.), number of training samples, and environmental scenario. Recently, one such public dataset named 'UWB-Gestures' has been presented in [125] for hand gesture recognition. It is the first public dataset of twelve dynamic hand gestures acquired with three ultra-wideband impulse radars.

As discussed in Section 5, dataset creation for micro-Doppler-based target recognition is a cumbersome task. Radar-based data gathering requires expensive equipment and extensive labour. In order to overcome the scarcity of training data, several solutions have been proposed in the literature [41]. These include generating synthetic micro-Doppler data from model based simulations, using GANs, unsupervised pre-training and transfer learning. However, none of these is the generalized solution valid for all sorts of target scenarios [41].

Micro-Doppler signature is a multi-component signal formed by the superposition of constituent mono-component signals. The decomposition of a microDoppler signature into components associated with individual structural parts of a target is still a challenge. Methods such as EMD can decompose a multicomponent micro-Doppler signal into constituent mono-components. However, these decomposed signals have no association with any structural part of the target. An effective decomposition algorithm that can perform physical component based decomposition of micro-Doppler signature will lead to improvement in target classification, recognition, and identification. It may also help in identifying and predicting the behaviour, emotions, and intentions of human targets [4].

Polarimetric micro-Doppler analysis can also aid in separation of different components of human motion. The double bounce of radar signal from dihedral joints like elbow and knees, can be separated from planar body surfaces by measuring the phase difference between $\mathrm{HH}$ and VV $[126,127]$. Use of dual polarization radar can be used to improve the classification accuracy for body gesture recognition, by combining the micro-Doppler signatures obtained using $\mathrm{HH}$ and HV [128]. Different types of polarimetric parameters have been used in [129] for classification of UAVs and birds using a S-band quadrature polarization radar.

Use of multistatic radars for capturing micro-Doppler returns is another research avenue that needs further exploration. A multistatic radar has spatially diverse transmitter / receiver nodes having a shared coverage area [130]. Use of multistatic radars offer certain advantages over monostatic radars, which include improvement in target detection, enhanced information about the target due to multiple aspect viewing, reduced vulnerability of the receiver to jamming, wider coverage, and use of clutter tuning for increased sensitivity [41,131,132]. 
Multistatic micro-Doppler signature is dependent on system topology, and the motion and location of the target [1]. By using the micro-Doppler information received from multiple channels, radar-based target classification can be improved as indicated in $[50,52,133,134]$.

The micro-Doppler signatures may be communicated to an operator or classification algorithm for a human-like experience. This can be in the form of aural signals or any other visual representation. Aural signal classification has been employed in sonar signal classification [135]. Advantage of aural classification is the robustness of human auditory classification process to noise. An audio depiction of micro-Doppler signal may help a human listener to differentiate between different human movements or between different target types [20]. Aural classification can be applied to micro-Doppler signatures by conversion of baseband micro-Doppler signal into an audio signal for training listeners [4]. Work on meaningful visual depictions of micro-Doppler data is also important for conveying useful information to the operator. Visual depiction can take the form of range-Doppler movies, as the processing speed has now improved to near real-time [136-138].

Another important research trend is augmenting micro-Doppler information with other forms of information from radar data, such as range, velocity, height, and direction of arrival information. With the development on millimeter wave radars with several $\mathrm{GHz}$ of bandwidth, returns from multiple targets and various parts of an object can be resolved in range. Use of micro-range micro-Doppler features can improve the classification performance and can also help in mapping motion components to individual body parts [41]. In [19], height, RCS, and velocity information is used in conjunction with micro-Doppler to achieve robust drone classification. In [139], micro-range micro-Doppler has been used to differentiate a single person from a group of people walking towards radar. Micro-Doppler and range-Doppler analysis has been used in [140] for detection of potential active shooter.

Wrong predictions in a real-time radar classification system can result in catastrophic outcomes. For example, confusing a bird with a drone can mistakenly trigger the air defense system in case of a military scenario. Hence, with regards to the classification approaches discussed in Section 4, there is a need to take into account the factors of data bias and uncertainty, as these approaches are data-driven. Is our training dataset labelled properly? Is our training dataset diverse enough to be free from data bias? How confident our classifier is about its predictions? Can we trust the predictions of our classifier on unseen data? Current work on micro-Doppler-based classification have not addressed these important questions.

\section{CONCLUSION}

With ever increasing applications of radar for remote sensing applications, there is a need for classification and recognition of different types of radar targets. Micro-Doppler signatures have emerged as the popular choice for classification 
of a wide range of air, ground, and space targets. Joint time-frequency techniques are used for micro-Doppler signature analysis since the spectral content of these signals vary with time. Linear time-frequency transforms have a tradeoff between time and frequency resolution while bilinear transforms suffer from the phenomenon of cross-term interference. DCNNs directly use micro-Doppler signature images as inputs for classification while conventional ML based classifiers require manual feature extraction step using different methods like EMD, SVD, PCA or ICA. Deep learning classifiers offer inherent advantages over conventional ML classifiers, but they require large and diverse training datasets for better classification performance. Micro-Doppler effect has found numerous applications in various fields like military, automotive industry, health sector, home automation and security, search and rescue, vital sign monitoring, and clutter rejection from wind turbines. There is a need of further work in this area, specifically in creation of standardized datasets by adopting the best practices, effective micro-Doppler signal decomposition, polarimetric and multistatic micro-Doppler analysis, optimization of feature extraction methods, addressing issues of uncertainty, and continual learning for practical systems.

\section{References}

[1] Graeme Edward Smith, "Radar target micro-Doppler signature classification," Ph.D. dissertation, University of London, 2008.

[2] S. S. Swords, Technical History of the Beginnings of Radar, ser. IEE history of technology series. London: Peregrinus on behalf of the Institution of Electrical Engineers, 1986, vol. 6.

[3] M. I. Skolnik, Introduction to Radar Systems. McGraw-hill New York, 1980 .

[4] V. C. Chen, The Micro-Doppler Effect in Radar, 2nd ed., ser. Artech House radar series. Norwood MA: Artech House, 2019.

[5] M. S. Zediker, R. R. Rice, and J. H. Hollister, "Method for extending range and sensitivity of a fiber optic micro-Doppler LADAR system and apparatus therefor," Dec. 8 1998, US Patent 5,847,817.

[6] C. Lei and Y. Ding, "Legs components micro-Doppler estimation of human target based on doppler radar," Journal of Physics: Conference Series, vol. 1544, p. 012044, may 2020. [Online]. Available: https://doi.org/10.1088/1742-6596/1544/1/012044

[7] R. I. Harmanny, J. J. de Wit, and G. Premel-Cabic, "Radar micro-Doppler mini-UAV classification using spectrograms and cepstrograms," International Journal of Microwave and Wireless Technologies, vol. 7, no. 3-4, pp. $469-477,2015$. 
[8] V. C. Chen, "Micro-Doppler effect of micromotion dynamics: A review," in Independent Component Analyses, Wavelets, and Neural Networks, vol. 5102. International Society for Optics and Photonics, 2003, pp. 240-249.

[9] Z. Liu and H.-x. Sun, "Micro-Doppler analysis and application of radar targets," in 2008 International Conference on Information and Automation. IEEE, 2008, pp. 1343-1347.

[10] V. C. Chen, "Advances in applications of radar micro-Doppler signatures," in 2014 IEEE Conference on Antenna Measurements \& Applications (CAMA). IEEE, 11/16/2014-11/19/2014, pp. 1-4.

[11] D. Tahmoush, "Review of micro-Doppler signatures," IET Radar, Sonar Es Navigation, vol. 9, no. 9, pp. 1140-1146, 2015.

[12] S. B. S. Hanbali, "A review of radar signals in terms of doppler tolerance, time-sidelobe level, and immunity against jamming," International Journal of Microwave and Wireless Technologies, vol. 10, no. 10, pp. 1134$1142,2018$.

[13] N. E. Huang, Z. Shen, S. R. Long, M. C. Wu, H. H. Shih, Q. Zheng, N.-C. Yen, C. C. Tung, and H. H. Liu, "The empirical mode decomposition and the Hilbert spectrum for nonlinear and non-stationary time series analysis," Proceedings of the Royal Society of London. Series A: mathematical, physical and engineering sciences, vol. 454, no. 1971, pp. 903-995, 1998.

[14] V. C. Chen, F. Li, S.-S. Ho, and H. Wechsler, "Micro-Doppler effect in radar: Phenomenon, model, and simulation study," IEEE Transactions on Aerospace and electronic systems, vol. 42, no. 1, pp. 2-21, 2006.

[15] J.-D. Wu and P.-H. Chiang, "Application of Wigner-Ville distribution and probability neural network for scooter engine fault diagnosis," Expert Systems with Applications, vol. 36, no. 2, Part 1, pp. 2187-2199, 2009. [Online]. Available: https://www.sciencedirect.com/science/article/pii/S0957417407006458

[16] S. Björklund, T. Johansson, and H. Petersson, "Evaluation of a microDoppler classification method on mm-wave data," in 2012 IEEE Radar Conference, 2012, pp. 0934-0939.

[17] A. M. Noll and M. R. Schroeder, "Short-time "cepstrum" pitch detection," The Journal of the Acoustical Society of America, vol. 36, no. 5, p. 1030, 1964. [Online]. Available: https://doi.org/10.1121/1.2143271

[18] X. Guo, C. S. Ng, E. de Jong, and A. B. Smits, "Micro-doppler based mini-uav detection with low-cost distributed radar in dense urban environment," in 2019 16th European Radar Conference (EuRAD), 2019, pp. 189-192. 
[19] M. Jahangir, B. I. Ahmad, and C. J. Baker, "Robust drone classification using two-stage decision trees and results from SESAR SAFIR trials," in 2020 IEEE International Radar Conference (RADAR). IEEE, 2020, pp. 636-641.

[20] V. C. Chen, D. Tahmoush, and W. J. Miceli, Radar micro-Doppler Signatures: Processing and Applications, ser. IET radar, sonar and navigation series. Stevenage: Institution of Engineering and Technology, 2014, vol. volume 34 .

[21] S. Rahman and D. A. Robertson, "Classification of drones and birds using convolutional neural networks applied to radar micro-Doppler spectrogram images," IET Radar, Sonar $\&$ Navigation, vol. 14, no. 5, pp. 653-661, 2020.

[22] Y. Kim and T. Moon, "Human detection and activity classification based on micro-Doppler signatures using deep convolutional neural networks," IEEE Geoscience and Remote Sensing Letters, vol. 13, no. 1, pp. 8-12, 2016.

[23] B. Choi and D. Oh, "Classification of drone type using deep convolutional neural networks based on micro-Doppler simulation," in 2018 International Symposium on Antennas and Propagation (ISAP). IEEE, 2018, pp. 1-2.

[24] B. K. Kim, H.-S. Kang, and S.-O. Park, "Drone classification using convolutional neural networks with merged Doppler images," IEEE Geoscience and Remote Sensing Letters, vol. 14, no. 1, pp. 38-42, 2017.

[25] J. Park, J.-S. Park, and S.-O. Park, "Small drone classification with light $\mathrm{CNN}$ and new micro-Doppler signature extraction method based on a-spc technique," arXiv preprint arXiv:2009.14422, 2020.

[26] J. J. De Wit, R. I. Harmanny, and P. Molchanov, "Radar micro-Doppler feature extraction using the singular value decomposition," in 2014 International Radar Conference. IEEE, 2014, pp. 1-6.

[27] L. Zhu, H. Zhao, H. Xu, X. Lu, S. Chen, and S. Zhang, "Classification of ground vehicles based on micro-Doppler effect and singular value decomposition," in 2019 IEEE Radar Conference (RadarConf), 2019, pp. $1-6$.

[28] A. Miller, C. Clemente, A. Robinson, D. Greig, A. Kinghorn, and J. Soraghan, "Micro-Doppler based target classification using multi-feature integration," in IET Intelligent Signal Processing Conference (ISP). IET, 2013. 
[29] Y. Yang, J. Lei, W. Zhang, and C. Lu, "Target classification and pattern recognition using micro-Doppler radar signatures," in Seventh ACIS International Conference on Software Engineering, Artificial Intelligence, Networking, and Parallel/Distributed Computing (SNPD'06). IEEE, 2006, pp. 213-217.

[30] X. Ma, B.-S. Oh, L. Sun, K.-A. Toh, and Z. Lin, "EMD-based entropy features for micro-Doppler mini-UAV classification," in 2018 24th International Conference on Pattern Recognition (ICPR). IEEE, 2018, pp. 1295-1300.

[31] B.-S. Oh, X. Guo, and Z. Lin, "A UAV classification system based on FMCW radar micro-Doppler signature analysis," Expert Systems with Applications, vol. 132, pp. 239-255, 2019.

[32] B.-S. Oh, X. Guo, F. Wan, K.-A. Toh, and Z. Lin, "Micro-Doppler miniUAV classification using empirical mode decomposition features," IEEE Geoscience and Remote Sensing Letters, vol. 15, no. 2, pp. 227-231, 2018.

[33] L. Fuhrmann, O. Biallawons, J. Klare, R. Panhuber, R. Klenke, and J. Ender, "Micro-Doppler analysis and classification of UAVs at Ka band," in 2017 18th International Radar Symposium (IRS), 2017, pp. 1-9.

[34] R. G. Raj, V. C. Chen, and R. Lipps, "Analysis of radar human gait signatures," IET Signal Processing, vol. 4, no. 3, pp. 234-244, 2010.

[35] R. G. Raj, V. C. Chen, and R. Lipps, "Analysis of radar dismount signatures via non-parametric and parametric methods," in 2009 IEEE Radar Conference, 2009, pp. 1-6.

[36] P. Li, D.-C. Wang, and L. Wang, "Separation of micro-Doppler signals based on time frequency filter and Viterbi algorithm," Signal, Image and Video Processing, vol. 7, no. 3, pp. 593-605, 2013.

[37] R. I. A. Harmanny, J. J. M. de Wit, and G. P. Cabic, Eds., Radar microDoppler feature extraction using the spectrogram and the cepstrogram.

[38] S. Björklund, H. Petersson, and G. Hendeby, "Features for micro-doppler based activity classification," IET radar, sonar $\& 3$ navigation, vol. 9, no. 9, pp. 1181-1187, 2015.

[39] A. K. Seifert, M. G. Amin, and A. M. Zoubir, "Toward unobtrusive in-home gait analysis based on radar micro-Doppler signatures," IEEE Transactions on Biomedical Engineering, vol. 66, no. 9, pp. 2629-2640, 2019.

[40] B. Çağllyan and S. Z. Gürbüz, "Micro-Doppler-based human activity classification using the mote-scale BumbleBee radar," IEEE Geoscience and Remote Sensing Letters, vol. 12, no. 10, pp. 2135-2139, 2015. 
[41] F. Fioranelli, H. Griffiths, M. Ritchie, and A. Balleri, Micro-Doppler Radar and its Applications. SciTech Publishing, 2020.

[42] P. Molchanov, "Radar target classification by micro-Doppler contributions," Ph.D. dissertation, Tampere University of Technology, 2014.

[43] R. Boulic, N. M. Thalmann, and D. Thalmann, "A global human walking model with real-time kinematic personification," The visual computer, vol. 6 , no. 6, pp. 344-358, 1990.

[44] A. Ghaleb, L. Vignaud, and J. Nicolas, "Micro-Doppler analysis of wheels and pedestrians in ISAR imaging," IET Signal Processing, vol. 2, no. 3, pp. 301-311, 2008.

[45] S. R. Groot, A. G. Yarovoy, R. I. A. Harmanny, and J. N. Driessen, "Model-based classification of human motion: Particle filtering applied to the micro-Doppler spectrum," in 2012 9th European Radar Conference, 2012, pp. 198-201.

[46] G. E. Smith, K. Woodbridge, and C. J. Baker, "Micro-Doppler signature classification," in 2006 CIE International Conference on Radar, 2006, pp. $1-4$.

[47] — - "Radar micro-Doppler signature classification using dynamic time warping," IEEE Transactions on Aerospace and Electronic Systems, vol. 46, no. 3, pp. 1078-1096, 2010.

[48] J. Lei and C. Lu, "Target classification based on micro-Doppler signatures," in IEEE International Radar Conference, 2005. IEEE, 2005, pp. $179-183$.

[49] P. Molchanov, K. Egiazarian, J. Astola, R. I. Harmanny, and J. J. de Wit, "Classification of small UAVs and birds by micro-Doppler signatures," in 2013 European Radar Conference. IEEE, 2013, pp. 172-175.

[50] F. Fioranelli, M. Ritchie, H. Griffiths, and H. Borrion, "Classification of loaded/unloaded micro-drones using multistatic radar," Electronics Letters, vol. 51, no. 22, pp. 1813-1815, 2015.

[51] J. Ren and X. Jiang, "Regularized 2-D complex-log spectral analysis and subspace reliability analysis of micro-Doppler signature for UAV detection," Pattern Recognition, vol. 69, pp. 225-237, 2017.

[52] F. Fioranelli, M. Ritchie, and H. Griffiths, "Multistatic human microDoppler classification of armed/unarmed personnel," IET Radar, Sonar \&3 Navigation, vol. 9, no. 7, pp. 857-865, 2015.

[53] J. Sim, M. Jahangir, F. Fioranelli, C. J. Baker, and H. Dale, "Effective ground-truthing of supervised machine learning for drone classification," in 2019 International Radar Conference (RADAR). IEEE, 2019, pp. 1-5. 
[54] M. S. Seyfioğlu, A. M. Özbayoğlu, and S. Z. Gürbüz, "Deep convolutional autoencoder for radar-based classification of similar aided and unaided human activities," IEEE Transactions on Aerospace and Electronic Systems, vol. 54, no. 4, pp. 1709-1723, 2018.

[55] G. Klarenbeek, R. I. A. Harmanny, and L. Cifola, "Multi-target human gait classification using LSTM recurrent neural networks applied to microDoppler," in 2017 European Radar Conference (EURAD), 2017, pp. 167170.

[56] X. Zhang, V. Mehta, M. Bolic, and I. Mantegh, "Hybrid AI-enabled method for UAS and bird detection and classification," in 2020 IEEE International Conference on Systems, Man, and Cybernetics (SMC), 2020, pp. 2803-2807.

[57] J. Zhu, H. Chen, and W. Ye, "Classification of human activities based on radar signals using 1d-CNN and LSTM," in 2020 IEEE International Symposium on Circuits and Systems (ISCAS), 2020, pp. 1-5.

[58] A. Shrestha, H. Li, J. Le Kernec, and F. Fioranelli, "Continuous human activity classification from FMCW radar with bi-LSTM networks," IEEE Sensors Journal, vol. 20, no. 22, pp. 13607-13619, 2020.

[59] Y. Sun, S. Abeywickrama, L. Jayasinghe, C. Yuen, J. Chen, and M. Zhang, "Micro-Doppler signature-based detection, classification, and localization of small UAV with long short-term memory neural network," IEEE Transactions on Geoscience and Remote Sensing, pp. 1-16, 2020.

[60] S. Hochreiter and J. Schmidhuber, "Long short-term memory," Neural computation, vol. 9, no. 8, pp. 1735-1780, 1997.

[61] A. Angelov, A. Robertson, R. Murray-Smith, and F. Fioranelli, "Practical classification of different moving targets using automotive radar and deep neural networks," IET Radar, Sonar $\&$ Navigation, vol. 12, no. 10, pp. 1082-1089, 2018.

[62] Q. Wu, T. Gao, Z. Lai, and D. Li, "Hybrid SVM-CNN classification technique for human-vehicle targets in an automotive LFMCW radar," Sensors, vol. 20, no. 12, 2020. [Online]. Available: https://www.mdpi.com/1424-8220/20/12/3504

[63] J. J. M. de Wit, R. I. A. Harmanny, and G. Prémel-Cabic, "Micro-Doppler analysis of small UAVs," in 2012 9th European Radar Conference, 2012, pp. 210-213.

[64] G. E. Hinton and R. R. Salakhutdinov, "Reducing the dimensionality of data with neural networks," science, vol. 313, no. 5786, pp. 504-507, 2006. 
[65] M. S. Seyfioğlu and S. Z. Gürbüz, "Deep neural network initialization methods for micro-Doppler classification with low training sample support," IEEE Geoscience and Remote Sensing Letters, vol. 14, no. 12, pp. 2462-2466, 2017.

[66] A. Miller, C. Clemente, A. Robinson, D. Greig, A. Kinghorn, and J. Soraghan, "Micro-Doppler based target classification using multi-feature integration," in IET Intelligent Signal Processing Conference 2013 (ISP2013). London, U.K.: IET, December 2-3, 2013.

[67] A. Kinghorn and A. Nejman, "PicoSAR- an advanced lightweight SAR system," in 2009 European Radar Conference (EuRAD), 2009, pp. 168171.

[68] R. Ricci and A. Balleri, "Recognition of humans based on radar microDoppler shape spectrum features," IET Radar, Sonar \& Navigation, vol. 9, no. 9, pp. 1216-1223, 2015.

[69] I. Alnujaim, D. Oh, and Y. Kim, "Generative adversarial networks for classification of micro-Doppler signatures of human activity," IEEE Geoscience and Remote Sensing Letters, vol. 17, no. 3, pp. 396-400, 2020.

[70] W. Zhang, G. Li, and C. Baker, "Radar recognition of multiple microdrones based on their micro-Doppler signatures via dictionary learning," IET Radar, Sonar $\& 5$ Navigation, vol. 14, no. 9, pp. 1310-1318, 2020.

[71] S. Rahman and D. A. Robertson, "Radar micro-Doppler signatures of drones and birds at K-band and W-band," Scientific reports, vol. 8, no. 1, pp. 1-11, 2018.

[72] X. Shi, Y. Li, F. Zhou, and L. Liu, "Human activity recognition based on deep learning method," in 2018 International Conference on Radar (RADAR). IEEE, 2018, pp. 1-5.

[73] Y. Yang, C. Hou, Y. Lang, D. Guan, D. Huang, and J. Xu, "Openset human activity recognition based on micro-Doppler signatures," Pattern Recognition, vol. 85, pp. 60-69, 2019. [Online]. Available: https://www.sciencedirect.com/science/article/pii/S003132031830270X

[74] M. McCloskey and N. J. Cohen, "Catastrophic interference in connectionist networks: The sequential learning problem," in Psychology of learning and motivation. Elsevier, 1989, vol. 24, pp. 109-165.

[75] D. Lee, H. Park, T. Moon, and Y. Kim, "Continual learning of microDoppler signature-based human activity classification," IEEE Geoscience and Remote Sensing Letters, pp. 1-5, 2021.

[76] J. Kirkpatrick, R. Pascanu, N. Rabinowitz, J. Veness, G. Desjardins, A. A. Rusu et al., "Overcoming catastrophic forgetting in neural networks," Proceedings of the national academy of sciences, vol. 114, no. 13, pp. 3521-3526, 2017. 
[77] F. Zenke, B. Poole, and S. Ganguli, "Continual learning through synaptic intelligence," in International Conference on Machine Learning. PMLR, 2017, pp. 3987-3995.

[78] R. Aljundi, F. Babiloni, M. Elhoseiny, M. Rohrbach, and T. Tuytelaars, "Memory aware synapses: Learning what (not) to forget," in Proceedings of the European Conference on Computer Vision (ECCV), 2018, pp. 139154.

[79] S.-A. Rebuffi, A. Kolesnikov, G. Sperl, and C. H. Lampert, "iCaRL: Incremental classifier and representation learning," in Proceedings of the IEEE conference on Computer Vision and Pattern Recognition, 2017, pp. 2001-2010.

[80] D. Lopez-Paz and M. Ranzato, "Gradient episodic memory for continual learning," arXiv preprint arXiv:1706.08840, 2017.

[81] A. Chaudhry, M. Rohrbach, M. Elhoseiny, T. Ajanthan, P. K. Dokania, P. H. Torr, and M. Ranzato, "On tiny episodic memories in continual learning," arXiv preprint arXiv:1902.10486, 2019.

[82] R. Petervari, F. Giovanneschi, W. Johannes, and M. González-Huici, "A realtime micro-Doppler detection, tracking and classification system for the 94 ghz FMCW radar system DUSIM," in 2019 16th European Radar Conference (EuRAD). IEEE, 2019, pp. 193-196.

[83] T. Wagner, R. Feger, and A. Stelzer, "Radar signal processing for jointly estimating tracks and micro-Doppler signatures," IEEE Access, vol. 5, pp. 1220-1238, 2017.

[84] S. Björklund, T. Johansson, and H. Petersson, "Target classification in perimeter protection with a micro-Doppler radar," in 2016 17th International Radar Symposium (IRS). IEEE, 2016, pp. 1-5.

[85] K. Olsen, T. Johnsen, S. Johnsrud, I. Tansem, and P. Sornes, "Multistatic and/or quasi monostatic radar measurements of propeller aircrafts," in 2007 IET International Conference on Radar Systems. IET, 2007, pp. $1-6$.

[86] T. Johnsen, K. Olsen, and R. Gundersen, "Hovering helicopter measured by bi-/multistatic CW radar," in Proceedings of the 2003 IEEE Radar Conference (Cat. No. 03CH37474). IEEE, 2003, pp. 165-170.

[87] P. Molchanov, A. Totsky, J. Astola, K. Egiazarian, S. Leshchenko, and M. Rosa-Zurera, "Aerial target classification by micro-Doppler signatures and bicoherence-based features," in 2012 9th European Radar Conference. IEEE, 2012, pp. 214-217. 
[88] A. Cilliers and W. Nel, "Helicopter parameter extraction using joint timefrequency and tomographic techniques," in 2008 International Conference on Radar. IEEE, 2008, pp. 598-603.

[89] Aveillant website. Available at https://www.aveillant.com/products/ (accessed Mar. 29,2021).

[90] L. Rashid and A. Brown, "Impact modelling of wind farms on marine navigational radar," in IET International Conference on Radar Systems, Edinburgh, U.K., October 5-18, 2007.

[91] G. J. Poupart, "Wind farms impact on radar aviation interests-final report," Sep 2003.

[92] R. J. Vogt, J. Reed, T. Crum, J. Snow, R. Palmer, B. Isom, and D. Burgess, "Impacts of wind farms on WSR-88D operations and policy considerations," in Preprints, 23rd Int. Conf. on Interactive Information Processing Systems (IIPS) for Meteorology, Oceanography, and Hydrology, San Antonio, TX, Amer. Meteor. Soc., Paper B, vol. 5, 2007.

[93] R. J. Vogt, "An update on policy considerations of wind farm impacts on WSR-88D systems," in 24th Conference on IIPS, 2008.

[94] F. Kong, Y. Zhang, and R. Palmer, "Wind turbine clutter mitigation for weather radar by adaptive spectrum processing," in 2012 IEEE Radar Conference, 2012, pp. 0471-0474.

[95] F. Kong, Y. Zhang, and R. Palmer, "Characterization of micro-Doppler radar signature of commercial wind turbines," in Radar Sensor Technology XVIII, vol. 9077. International Society for Optics and Photonics, 2014, p. 907714 .

[96] O. A. Krasnov and A. G. Yarovoy, "Radar micro-Doppler of wind turbines: Simulation and analysis using rotating linear wire structures," International Journal of Microwave and Wireless Technologies, vol. 7, no. 3-4, pp. 459-467, 2015.

[97] R. Nepal, J. Cai, and Z. Yan, "Micro-Doppler radar signature identification within wind turbine clutter based on short-cpi airborne radar observations," IET Radar, Sonar \& Navigation, vol. 9, no. 9, pp. 1268-1275, 2015 .

[98] R. Kassab, G. G. de Charnace, and C. Adnet, "Classification of the wind turbine generated radar detections by artificial intelligence," in 2019 International Radar Conference (RADAR), 2019, pp. 1-2.

[99] A. R. Persico, C. Clemente, D. Gaglione, C. V. Ilioudis, J. Cao, L. Pallotta, A. De Maio, I. Proudler, and J. J. Soraghan, "On model, algorithms, and experiment for micro-doppler-based recognition of ballistic targets," IEEE Transactions on Aerospace and Electronic Systems, vol. 53, no. 3, pp. 1088-1108, 2017. 
[100] H. Gao, L. Xie, S. Wen, and Y. Kuang, "Micro-Doppler signature extraction from ballistic target with micro-motions," IEEE Transactions on Aerospace and Electronic Systems, vol. 46, no. 4, pp. 1969-1982, 2010.

[101] I. Choi, S. Park, M. Kim, K. Kang, and K. Kim, "Efficient discrimination of ballistic targets with micromotions," IEEE Transactions on Aerospace and Electronic Systems, vol. 56, no. 2, pp. 1243-1261, 2020.

[102] X. Zou, L. Gao, Q. Chen, and J. Yu, "Parameter estimation of the precessing cone-shaped warhead based on the micro-Doppler with multi-aspect observations," IOP Conference Series: Materials Science and Engineering, vol. 274, p. 012027, 2017.

[103] L. Han, C. Feng, and L. Luini, "Micro-Doppler-based space target recognition with a one-dimensional parallel network," International Journal of Antennas and Propagation, vol. 2020, p. 8013802, 2020.

[104] Y. Wang, C. Feng, Y. Zhang, and Q. Ge, "Classification of space targets with micro-motion based on deep CNN," in 2019 IEEE 2nd International Conference on Electronic Information and Communication Technology (ICEICT), 2019, pp. 557-561.

[105] Y. Zhou, Z. Chen, L. Zhang, and J. Xiao, "Micro-Doppler curves extraction and parameters estimation for cone-shaped target with occlusion effect," IEEE Sensors Journal, vol. 18, no. 7, pp. 2892-2902, 2018.

[106] S. Yuchun, J. Bo, L. Yuan, and L. Hongwei, "A coning micro-Doppler signals separation algorithm based on time-frequency information," in 2017 IEEE International Conference on Signal Processing, Communications and Computing (ICSPCC), 2017, pp. 1-5.

[107] L. Liu, Des McLernon, M. Ghogho, W. Hu, and J. Huang, "Ballistic missile detection via micro-Doppler frequency estimation from radar return," Digital Signal Processing, vol. 22, no. 1, pp. 87-95, 2012.

[108] Y. Kim and B. Toomajian, "Hand gesture recognition using micro-Doppler signatures with convolutional neural network," IEEE Access, vol. 4, pp. 7125-7130, 2016.

[109] G. Li, R. Zhang, M. Ritchie, and H. Griffiths, "Sparsity-driven microDoppler feature extraction for dynamic hand gesture recognition," IEEE Transactions on Aerospace and Electronic Systems, vol. 54, no. 2, pp. 655-665, 2018.

[110] M. G. Amin, Z. Zeng, and T. Shan, "Hand gesture recognition based on radar micro-Doppler signature envelopes," in 2019 IEEE Radar Conference (RadarConf), 2019, pp. 1-6. 
[111] G. Li, R. Zhang, M. Ritchie, and H. Griffiths, "Sparsity-based dynamic hand gesture recognition using micro-Doppler signatures," in 2017 IEEE Radar Conference (RadarConf), 2017, pp. 0928-0931.

[112] Z. Chen, G. Li, F. Fioranelli, and H. Griffiths, "Dynamic hand gesture classification based on multistatic radar micro-Doppler signatures using convolutional neural network," in 2019 IEEE Radar Conference (RadarConf), 2019, pp. 1-5.

[113] U.S. Center for Disease Control and Prevention website. Available at https://www.cdc.gov/falls/facts.html (accessed Apr. 1,2021).

[114] M. Wu, X. Dai, Y. D. Zhang, B. Davidson, M. G. Amin, and J. Zhang, "Fall detection based on sequential modeling of radar signal timefrequency features," in 2013 IEEE International Conference on Healthcare Informatics, 2013, pp. 169-174.

[115] B. Jokanovic, M. Amin, F. Ahmad, and B. Boashash, "Radar fall detection using principal component analysis," in Radar Sensor Technology XX, K. I. Ranney and A. Doerry, Eds., vol. 9829, International Society for Optics and Photonics. SPIE, 2016, pp. 358 - 363. [Online]. Available: https://doi.org/10.1117/12.2225106

[116] B. Jokanović and M. Amin, "Fall detection using deep learning in rangeDoppler radars," IEEE Transactions on Aerospace and Electronic Systems, vol. 54, no. 1, pp. 180-189, 2018.

[117] S. Z. Gurbuz, C. Clemente, A. Balleri, and J. J. Soraghan, "Micro-dopplerbased in-home aided and unaided walking recognition with multiple radar and sonar systems," IET Radar, Sonar $\&$ Navigation, vol. 11, no. 1, pp. 107-115, 2016.

[118] A.-K. Seifert, L. Schäfer, M. G. Amin, and A. M. Zoubir, "Subspace classification of human gait using radar micro-doppler signatures," in 2018 26th European Signal Processing Conference (EUSIPCO), 2018, pp. 311315 .

[119] J. Salmi, O. Luukkonen, and V. Koivunen, "Continuous wave radar based vital sign estimation: Modeling and experiments," in 2012 IEEE Radar Conference, 2012, pp. 0564-0569.

[120] R. M. Narayanan, "Earthquake survivor detection using life signals from radar micro-Doppler," in Proceedings of the 1st International Conference on Wireless Technologies for Humanitarian Relief - ACWR '11, R. Chidambaram, P. V. Rangan, S. Tripathi, P. Mohapatra, M. V. Ramesh, and M. van Steen, Eds. ACM Press, 2011, p. 259.

[121] N. Regev and D. Wulich, "Remote sensing of vital signs using an ultra-wide-band radar," International Journal of Remote Sensing, vol. 40, no. 17, pp. 6596-6606, 2019. [Online]. Available: https://doi.org/10.1080/2150704X.2019.1573335 
[122] S. Shirodkar, P. Barua, D. Anuradha, and R. Kuloor, "Heart-beat detection and ranging through a wall using ultra wide band radar," in 2011 International Conference on Communications and Signal Processing, 2011, pp. 579-583.

[123] P. Chen, M. C. Shastry, C. Lai, and R. M. Narayanan, "A portable real-time digital noise radar system for through-the-wall imaging," IEEE Transactions on Geoscience and Remote Sensing, vol. 50, no. 10, pp. 41234134, 2012.

[124] L. Ren, N. Tran, H. Wang, A. E. Fathy, and O. Kilic, "Analysis of microDoppler signatures for vital sign detection using UWB impulse Doppler radar," in 2016 IEEE Topical Conference on Biomedical Wireless Technologies, Networks, and Sensing Systems (BioWireleSS), 2016, pp. 18-21.

[125] S. Ahmed, D. Wang, J. Park, and S. H. Cho, "Uwb-gestures, a public dataset of dynamic hand gestures acquired using impulse radar sensors," Scientific Data, vol. 8, no. 1, pp. 1-9, 2021.

[126] D. Tahmoush and J. Silvious, "Human polarimetric micro-Doppler," in Radar Sensor Technology XV, vol. 8021. International Society for Optics and Photonics, 2011, p. 802106.

[127] A. Freeman, "Fitting a two-component scattering model to polarimetric SAR data from forests," IEEE Transactions on Geoscience and Remote Sensing, vol. 45, no. 8, pp. 2583-2592, 2007.

[128] W. Kang, Y. Zhang, and X. Dong, "Body gesture recognition based on polarimetric micro-Doppler signature and using deep convolutional neural network," Progress In Electromagnetics Research, vol. 79, pp. 71-80, 2019.

[129] B. Torvik, K. E. Olsen, and H. Griffiths, "Classification of birds and UAVs based on radar polarimetry," IEEE Geoscience and Remote Sensing Letters, vol. 13, no. 9, pp. 1305-1309, 2016.

[130] V. S. Chernyak, Fundamentals of Multisite Radar Systems: Multistatic Radars and Multistatic Radar Systems. CRC press, 1998.

[131] S. Kingsley and S. Quegan, Understanding Radar Systems. SciTech Publishing, 1999, vol. 2.

[132] M. Inggs, H. Griffiths, F. Fioranelli, M. Ritchie, and K. Woodbridge, "Multistatic radar: system requirements and experimental validation," in 2014 International Radar Conference, 2014, pp. 1-6.

[133] F. Fioranelli, M. Ritchie, and H. Griffiths, "Aspect angle dependence and multistatic data fusion for micro-Doppler classification of armed/unarmed personnel," IET Radar, Sonar \& Navigation, vol. 9, no. 9, pp. 1231-1239, 2015 . 
[134] B. Tekeli, S. Z. Gürbüz, M. Yüksel, A. C. Gürbüz, and M. B. Guldogan, "Classification of human micro-Doppler in a radar network," in 2013 IEEE Radar Conference (RadarCon13), 2013, pp. 1-6.

[135] N. Allen, P. C. Hines, V. W. Young, and D. A. Caldwell, "Study on the human ability to aurally discriminate between target echoes and environmental clutter in recordings of incoherent broadband sonar," The Journal of the Acoustical Society of America, vol. 119, no. 5, pp. 3395-3395, 2006.

[136] Y. Wang, Q. Liu, and A. E. Fathy, "CW and pulse-Doppler radar processing based on FPGA for human sensing applications," IEEE Transactions on Geoscience and Remote Sensing, vol. 51, no. 5, pp. 3097-3107, 2013.

[137] V. Kozlov, E. Rebenshtok, and P. Ginzburg, "Synthetic software defined radar (conference presentation)," in Radar Sensor Technology XXIV, vol. 11408. International Society for Optics and Photonics, 2020, p. 114080G.

[138] M. J. Nazaroff, H.-J. Kong, and Y. Kim, "Tracking bedridden patient positions using micro-Doppler signatures," IEEE Sensors Letters, vol. 5, no. 4, pp. 1-4, 2021.

[139] D. Tahmoush, "Micro-range micro-Doppler for classification," in 2020 IEEE Radar Conference (RadarConf20), 2020, pp. 1-4.

[140] Y. Li, Z. Peng, R. Pal, and C. Li, "Potential active shooter detection based on radar micro-Doppler and range-Doppler analysis using artificial neural network," IEEE Sensors Journal, vol. 19, no. 3, pp. 1052-1063, 2019. 